\title{
Circadian rhythms: from genes to behaviour
}

\author{
Preface
}

It was not too long ago that the very mention of the phrase 'biological clock' generated cynical responses from biologists. Yet, today biological clocks are one of the best understood complex phenomena in biology. The main reason for the early scepticism was probably the close resemblance of these rhythms to many geophysical cycles, and the lack of clear evidence for their adaptive value. No doubt such criticism led to the field becoming more rigorous and logical. It forced people to conduct experiments to find out the genetic basis of clocks, leading to the discovery of clock genes, and their anatomical correlates the circadian pacemakers, and to study behaviours with direct adaptive implications such as leaf movement in plants, adult emergence rhythm in fruit flies and foraging in honeybees. This also motivated people to carry out experiments in environments where there would be little or no influence of geophysical and cosmic cycles. However, it was not until 1971 when Ron Konopka and Seymour Benzer identified the first clock gene period (per) in Drosophila melanogaster, which mapped on to the right arm of the X chromosome, that sceptics were won over. There has been no looking back ever since. Several clock genes have subsequently been identified in a wide range of organisms, and their roles in the molecular clockwork elucidated. Some other milestones in this journey were the identification and characterization of frequency ( frq), one of the core clock genes in the fungus Neurospora crassa by Jerry F. Feldman's group, timeless (tim) in D. melanogaster by Michael W. Young's group, and identification and characterization of CLOCK in mammals by Joseph Takahashi's group. Further work building on these findings led to the formulation of a functional model for the circadian clockwork based on a transcriptional-translational feedback loop, comprising of transcripts and proteins of several core clock genes. The clock receives sensory information in order to retain synchrony with local time, and communicates with the rest of the organism via a wide array of rhythmic output signals including PIGMENT DISPERSING FACTOR (PDF) in insects and VIP, AVP, and other neurotransmitters in mammals. While the molecular mechanisms of biological clocks were being explored in a few model organisms, the search for the location of pacemaker sites was also underway. Finally, the master circadian pacemaker in mammals was localized to the suprachiasmatic nucleus (SCN) located in the third ventricle of the hypothalamus by two independent groups: F. K. Stephan and Irvin Zucker, and Robert Y. Moore and V. B. Eichler in 1974. In D. melanogaster too, circadian pacemakers were localized in the lateral and dorsal part of the brain; a number of neuronal subgroups were identified to be the circadian pacemaker neurons, based on their expression of clock proteins and neurotransmitters. These findings gave a great boost to research in circadian neurobiology.

Chronobiology has made, and is still making, tremendous progress at breakneck speed. This success is primarily due to the integrative approach followed in this area, an approach that was a pivotal part of this discipline right from its inception. Organisms were observed in nature and under controlled laboratory conditions, leading to the formulation of hypotheses which, in turn, led to construction of models, and models led to standardized experiments which were then used to study a host of different organisms ranging from bacteria to humans. By 1960, when the first meeting of chronobiologists was held at Cold Spring Harbour, the field already knew where it was heading and what the essential questions were. Thus, a clear research goal and programme were in place that eventually resulted in an explosion of knowledge about circadian timing systems. Circadian biologists felt a need to move forward by incorporating different approaches in biology - ecological, behavioural, genetic, molecular, cellular, neuroendocrine and physiological, including studies of phenomena with obvious adaptive value such as photoperiodism, hibernation, and migration. Integrative studies on a few focal organisms provided us with a better understanding of molecular, cellular and organismal integration of the circadian machinery and its functioning.

Although the past three decades have witnessed enormous progress in understanding the molecular mechanisms of circadian rhythmicity, the field has not neglected other aspects of chronobiology. Thus, while some researchers have used newly devised tools in molecular genetics to discover more elements of the core clock mechanism and to understand the circadian clockwork at molecular and physiological levels, others continued to probe the key characteristics of circadian rhythms at the whole organism level-a true hallmark of an integrative approach in biology. 
Circadian rhythms are one of the most tractable phenomena in biology for which molecular phenomena in the cell can be connected to complex whole-organism behaviours. What is most remarkable is that rhythmic signals generated in the cells travel practically unperturbed all the way to their target organs that eventually regulate behaviour. This provides us with a unique opportunity to tap the signals at various levels of biological organization, and we have tried to represent this aspect of our discipline in this special issue of the Journal of Genetics. While the issue contains papers on hard-core molecular biology and genetics, such as those on the role of CLOCK protein, casein kinase I, and microRNAs in the regulation of circadian rhythms, genetic variability in clock genes, molecular interface between metabolic and clock genes and the neurogenetic basis of circadian rhythms, we have also included papers that highlight the role of circadian clocks in the regulation of behaviour. This was done to exemplify the inherently integrative nature of circadian rhythm research, because at the core of all these studies lies a genetic architecture which is responsible for driving rhythmic behaviour on a circadian time scale.

We hope that readers of this special issue will sense the spirit of excitement that permeates the field of circadian biology today: the papers included in this issue represent the breadth, scope and vigour of a field that is progressing at a remarkably rapid speed. With this special issue, we also register our deepest appreciation for three people who were instrumental in laying the foundation of the field of circadian biology: Erwin Bünning, Colin S. Pittendrigh, and Jurgen Aschoff, and for all those who are responsible for shaping it to its present form.

ViJay K. Sharma

ROBERT J. Lucas

Editors

E-mail: vsharma@jncasr.ac.in; robert.lucas@manchester.ac.uk. 